\title{
Impacto da suplementação de óleo de peixe rico em ácido graxo poli-insaturado ômega 3 sobre a função e morfologia renal de ratos Wistar submetidos à isquemia quente
}

\author{
Impact of oil fish rich in polyunsaturated fatty acids omega \\ 3 supplementation on renal function and morphology \\ in Wistar rats submitted to warm ischemia
}

Patrícia Regina Erdmann Mosko ${ }^{[a]}$,Fernando Meyer ${ }^{[b]}$,Flavio de Oliveira Lima ${ }^{[c]}$, Luiz Claudio Fernandes ${ }^{[d]}, J u l i a n y ~ G o m e s ~ Q u i t z a n n^{[e]}$

[a] Professora Assistente da Faculdade de Medicina Veterinária da Universidade Positivo (UP), Aluna do Programa de Pós-graduação em Ciência Animal da Pontifícia Universidade Católica do Paraná (PUCPR), Curitiba, PR, Brasil, e-mail: patricia1804@gmail.com

[b] Professor Titular da Faculdade de Medicina da Pontifícia Universidade Católica do Paraná (PUCPR), Curitiba, PR, Brasil, e-mail: fmeyer@urocentro.com

[c] Professor Assistente Doutor da Faculdade de Medicina da Universidade Estadual Paulista “Júlio de Mesquita Filho" (UNESP), Botucatu, SP, Brasil, e-mail: flavioolima@fmb.unesp.br

[d] Professor Titular da Universidade Federal do Paraná (UFPR), Curitiba, PR, Brasil, e-mail: lcfer@ufpr.br

[e] Professora Assistente Doutora da Faculdade de Medicina Veterinária e Zootecnia da Universidade Estadual Paulista "Júlio de Mesquita Filho" (UNESP), Botucatu, SP, Brasil, e-mail: juquitzan@fmvz.unesp.br

\section{Resumo}

A isquemia quente renal (IQR) realizada nas cirurgias de transplantes inter vivos e nefrectomias parciais pode contribuir para o desenvolvimento de injúria renal aguda e rejeição do órgão transplantado, na dependência da sua duração e da função renal pré-operatória. 0 uso dos ácidos graxos poli-insaturados ômega 3 tem sido alvo de pesquisas devido às suas propriedades anti-inflamatórias, antioxidantes, antifibróticas, vasodilatadoras e antitrombogênicas, com perspectivas promissoras nas lesões isquêmicas renais. Este estudo teve por objetivos comparar a função renal e alterações microscópicas renais em ratos Wistar, submetidos à IQR, suplementados com óleo de peixe enriquecido com ácido graxo poli-insaturado ômega-3. Os animais foram distribuídos aleatoriamente em quatro grupos ( $n=12$ ): (GC) sem suplementação, (G1) suplementação 30 dias antes da IQR, (G2) suplementação 30 dias após IQR, (G3) suplementação 30 dias antes e depois da IQR. Metade dos animais de cada grupo foi submetida à eutanásia após 24 horas e o restante após 30 dias do procedimento. Aos 30 dias após IQR, todos animais suplementados apresentaram elevação dos valores de creatinina plasmática, quando comparada à creatinina pré isquemia $(\mathrm{p}<0,05)$. Comparando os três grupos suplementados ao grupo controle, G2 apresentou creatinina plasmática superior no pós-operatório de 30 dias $(p<0,05)$. Não se observou diferença estatística nos escores histopatológicos renais entre os grupos. Conclui-se que, nas condições propostas, o uso de ácido graxo poli-insaturado ômega 3 em todos os grupos suplementados não evitou o aumento de creatinina plasmática após isquemia quente renal, entretanto preservou a morfologia renal.

Palavras-chave: Ácido graxo poli-insaturado ômega 3. Nefroproteção. Isquemia quente. Histopatologia renal. 


\section{Abstract}

The warm renal ischemia (WRI) performed in the surgeries of living kidney transplantation and partial nephrectomies may contribute to the development of acute renal injury and rejection of the transplanted organ, depending on its duration and preoperative renal function. The use of omega-3 polyunsaturated fatty acids has been the subject of research due to its anti-inflammatory, antioxidant, antifibrotic, vasodilating and antithrombogenic properties, with promising prospects for renal ischemic lesions. This study aimed to compare renal function and renal histopathology in Wistar rats, submitted to WRI, supplemented with fish oil enriched with omega-3 polyunsaturated fatty acid. The animals were randomly divided into 4 groups $(n=12):(C G)$ without supplementation; (G1) supplementation 30 days prior to WRI; (G2) supplementation 30 days after WRI; (G3) supplementation 30 days before and after WRI. Half of the animals in each group were euthanized after 24 hours and the remainder after 30 days of the procedure. At 30 days after WRI, all supplemented animals had increased plasma creatinine values, when compared to creatinine pre-ischemia $(p<0.05)$. Comparing the 3 groups supplemented to the control group, G2 presented higher plasma creatinine in the postoperative period of 30 days $(p<0.05)$. There was no statistical difference in the histopathological lesion scores between the groups. It was concluded that, under the proposed conditions, the use of omega-3 polyunsaturated fatty acids in all supplemented groups did not prevent an increase in plasma creatinine after warm renal ischemia, but preserved renal morphology.

Keywords: Omega-3 polyunsaturated fatty acids. Nephroprotection. Warm ischemia. Reperfusion. Renal histopathology.

\section{Introdução}

A isquemia quente com consequente interrupção momentânea do fluxo sanguíneo renal leva a importante redução da hemodinâmica e pode causar lesões morfofuncionais momentâneas ou permanentes do órgão (Dejavaran, 2006; Tennankore et al., 2016). No entanto, é etapa fundamental em alguns procedimentos cirúrgicos como nefrectomia parcial, nefrotomia e captação de rins de doadores vivos quentes (Halazun et al., 2007; Adin e Scansen, 2011; Holt, 2011; Tennankore et al., 2016). Quando as lesões tissulares são extensas a ponto de comprometerem cerca de $65 \%$ da massa funcional renal, estabelece-se a injúria renal aguda (IRA), que caracteriza-se como declínio abrupto da função renal, avaliada pela elevação sérica da creatinina basal ou redução do débito urinário (Ronco, 2016). Estas alterações ocorrem de horas a semanas após a injúria, contudo, em período inferior a três meses, com alta morbidade e mortalidade (Vaidya et al., 2008; Bonventre e Yang, 2011; Pieckering e Endre, 2014).
Aocorrência da IRA pós-cirúrgica em nefrectomia parcial foi relacionada com tempos de isquemia prolongados, sendo proporcional à presença de menor volume funcional de néfrons na massa renal remanescente (Thompson et al., 2010; Thompson et al., 2012; Abdeldaeim et al., 2015; Volpe et al., 2015). Tennankore et al. (2016) investigaram retrospectivamente a função precoce de rins transplantados e seus resultados corroboram com outros autores (Siedlecki et al., 2011; Hellegering et al., 2012), revelando que o prolongado tempo de isquemia quente está diretamente relacionado à diminuição da sobrevida do enxerto transplantado e ao óbito. Atribui-se ao procedimento de isquemia quente causa pré-renal (hemodinâmica) e renal intrínseca (isquemia e reperfusão) de lesão aguda e potencialmente permanente (Nolan e Anderson, 1998; Malek e Nematbakhsh, 2015).

0 reestabelecimento da perfusão tecidual é fundamental para minimizar tais danos, contudo, a circulação de radicais livres de oxigênio formados durante o período de isquemia inicia complexa cascata de eventos inflamatórios, levando ao 
acúmulo intracelular de cálcio (Evora et al., 1996; Malek e Nematbakhsh, 2015), apoptose e necrose celular (Padanilam, 2003).

Sabe-se que a via comum das lesões renais envolve inflamação e fibrose; desta forma, terapias que possam influenciar a resposta inflamatória vêm sendo estudadas, incluindo o uso de ácidos graxos poli-insaturados ômega 3 (AGPI n-3) como moduladores dos mediadores inflamatórios derivados de lipídios (Fassett et al., 2010; Hong e Lu, 2013).

A família ômega 3, representada especialmente pelo EPA (ácido eicosapentaenóico) e DHA (ácido docosahexaenóico), é composta por ácidos graxos essenciais, poli-insaturados, e tem especial importância na composição fosfolipídica da membrana plasmática (Waitzberg, 2009). Desempenham funções nutricional, estrutural e reguladora dos lipídios, atuando de forma significativa na hemodinâmica e oxigenação, assim como na resposta inflamatória e controle de triglicerídeos (Waitzberg, 2009). Ainda, a proteção celular é atribuída à ação inibitória sobre a produção de eicosanoides derivados do ácido araquidônico, como prostaglandinas, leucotrienos, tromboxanos e citocinas pró-inflamatórias nos tecidos periféricos (Waitzberg, 2009). Atuam também sobre a produção de resolvinas e na regulação da expressão gênica de fator de transcrição nuclear kapa B (NFkB) [um sinalizador inflamatório] e nos receptores ativados por proliferadores do peroxissoma (PPARs), que possuem ação anti-inflamatória (Yaqoob, 2009). Ressalta-se também seu efeito antitrombogênico (Woodman et al., 2003), culminando em menor agregação plaquetária.

O objetivo deste estudo foi avaliar a função e morfologia de rins de ratos Wistar submetidos à isquemia quente unilateral, submetidos à suplementação oral de óleo de peixe rico em AGPI n-3, em dois momentos pós-operatórios.

\section{Material e métodos}

0 presente trabalho foi aprovado pela Comissão de Ética no Uso de Animais (CEUA) da Pontifícia Universidade Católica do Paraná (PUCPR), sob número de registro 741/2012.
Foram utilizados 48 ratos Wistar (Rattus novergicus), machos, adultos, pesando entre $250 \mathrm{e}$ $400 \mathrm{~g}$ acondicionados em gaiolas com água e comida ad libitum, com temperatura $\left(23^{\circ} \mathrm{C}\right)$, umidade e ciclo dia e noite (12 horas) controlados. Todos os animais foram submetidos ao procedimento de isquemia quente renal (IQR) do rim esquerdo com clampe vascular atraumático por 30 minutos (Simmons et al., 2011), seguido de reperfusão e, imediatamente depois, nefrectomia do rim oposto (direito).

Os animais foram distribuídos aleatoriamente em quatro grupos $(n=12)$, conforme programa de suplementação oral de óleo de peixe comercial - Óleo de Peixe Herbarium ${ }^{\circledR}$, cápsulas de $1 \mathrm{~g}$ contendo 0,2g de EPA e 0,1g de DHA (EPA:DHA 2:1) - na dose de $1 \mathrm{~g} \cdot \mathrm{Kg}^{-1}$ : (GC) sem suplementação, (G1) suplementação prévia de 30 dias à IQR, (G2) suplementação posterior de 30 dias à IQR, (G3) suplementação prévia e posterior, ambas de 30 dias à IQR. Metade dos animais de cada grupo foi submetido à eutanásia em 24 horas pós procedimento de isquemia e reperfusão, sendo os demais submetidos à eutanásia 30 dias pós IQR. Os rins direitos dos animais pertencentes aos grupos GC e G2 foram utilizados como controle de normalidade sem suplementação.

A concentração plasmática de creatinina $\left(\right.$ LaborClin $^{\circledR}$ ) foi avaliada no pré-operatório imediato e no momento da eutanásia, em todos os animais.

Os rins foram fixados em solução de formaldeído a $4 \%$ por 24 horas e em seguida processados segundo histotecnologia de rotina para a obtenção de secções de $4 \mu \mathrm{m}$ de espessura. Os cortes foram corados com hematoxilina-eosina (HE) e analisados à microscopia de luz por patologista que desconhecia os protocolos de suplementação utilizados.

Foi definido como IRA o aumento pósoperatório de creatinina plasmática superior a 0,3 mg.dL ${ }^{-1}$ quando comparado com momento préoperatório imediato, conforme classificação RIFLE, demonstrada na Tabela 1.

Foram avaliados todos os rins isquemiados de todos os grupos (GC, G1, G2 e G3) e seis rins direitos, não isquemiados e não suplementados (do grupo controle). A análise histopatológica foi realizada adaptando-se aos critérios de Goujon et al. (1999), utilizando-se cinco critérios passíveis 
de observação à microscopia de luz: vacuolização citoplasmática apical, descolamento celular, necrose tubular, presença de material eosinofílico na luz do túbulo e infiltrado inflamatório, graduados em escores conforme a abragência das lesões: 1 nenhuma anormalidade, 2 - lesões afetando 10\% ou menos do rim, 3 - lesões afetando $25 \%$ do rim, 4 - lesões afetando $50 \%$ do rim e 5 - $75 \%$ ou mais de lesão renal. Os escores de cada critério foram somados em cada um dos animais, conferindo uma pontuação individual. A pontuação individual foi somada dentro dos grupos, conferindo uma pontuação global do grupo, tanto no momento 24 horas, quanto no momento 30 dias.

As análises foram realizadas utilizando o software estatístico GraphPad Prism version 3.00 for Windows, San Diego - Califórnia, EUA. Foi utilizado test $\mathrm{T}$ de student pareado para análise dentro dos grupos, análise de variância (One-way ANOVA) não pareado seguido pelo teste de médias de Bonferroni para análise entre grupos e teste de Kruskal-Wallis seguido pelo teste de médias de Dunn's para as variáveis não paramétricas. Para todas as análises foi adotado nível de significância de 5\% $(\mathrm{p}<0,05)$.

Tabela 1 - Sistema de classificação RIFLE para pacientes com IRA (Injúria Renal Aguda)

\begin{tabular}{ll}
\hline \multicolumn{1}{c}{ Estágio } & \multicolumn{1}{c}{ Creatinina Sérica } \\
\hline 1 - Risco “Risk" & Aumento de $\geqslant 0,3 \mathrm{mg} \cdot \mathrm{dL}^{-1}$ ou $\geqslant 150$ a $200 \%$ do valor basal \\
\hline 2 - Injúria "Injury" & Aumento de $200-300 \%$ do valor basal \\
\hline - Falência "Failure" & Aumento > 300\% do valor basal \\
\hline Perda “Loss" & IRA persistente, completa perda de função por 4 semanas \\
\hline Estágio Final “End-Stage" & Perda da função renal por 3 meses \\
\hline
\end{tabular}

Nota: Adaptado de Vaidya et al., 2008.

\section{Resultados}

A dosagem de creatinina feita após 24 horas na metade dos animais de cada grupo revelou que $75 \%$ destes (18/24; sendo GC: 5/6, G1: 4/6, G2: 5/6 e G3: 4/6) apresentaram aumento superior a $0,3 \mathrm{mg} \cdot \mathrm{dL}^{-1}$. Destes, apenas um animal (G1) apresentou aumento de creatinina basal superior a $300 \%$ e dois animais (GC e G2) apresentaram aumento de creatinina basal entre 200 e $300 \%$. Os demais (15/18) apresentaram aumento superior a $0,3 \mathrm{mg} \cdot \mathrm{dL}^{-1}$ e até de $200 \%$ do valor basal.

Na dosagem de creatinina realizada no momento da eutanásia aos 30 dias pós-IQR, observou-se que dos 24 animais avaliados, apenas dois apresentaram aumento de creatinina plasmática superior a 0,3 mg.dL $\mathrm{dL}^{-1}$ (um animal de cada grupo G2 e G3), equivalente a $8,3 \%$. Contudo, observou-se diferença entre as médias de creatinina pré e 30 dias pósIQR em todos os grupos suplementados, o que não aconteceu no grupo controle, conforme demonstra a Figura 1. Ainda, todas as médias pós-IQR 30 dias revelaram-se acima dos valores de normalidade para a espécie (VR 0,3-0,8mg.dL ${ }^{-1}$ ) (Branco et al., 2011), conforme Tabela 2.

Comparando-se os valores de creatinina plasmática pós-operatória no momento 30 dias entre os grupos, observa-se que há diferença estatística $(P=0,0283)$ entre GC x G2, conforme Figura 2.

À análise histopatológica, para cada critério avaliado, não foi observada diferença estatística entre os grupos analisados, comparando-se os grupos controle G1, G2, G3, e rins direitos normais (N) no momento 24 horas e 30 dias. Contudo, quando somados os escores de cada critério avaliado em todos os animais de cada grupo, a pontuação total obtida diferiu entre os rins isquemiados e os rins normais tanto no momento 24 horas, quanto no momento 30 dias (Figura 3). 


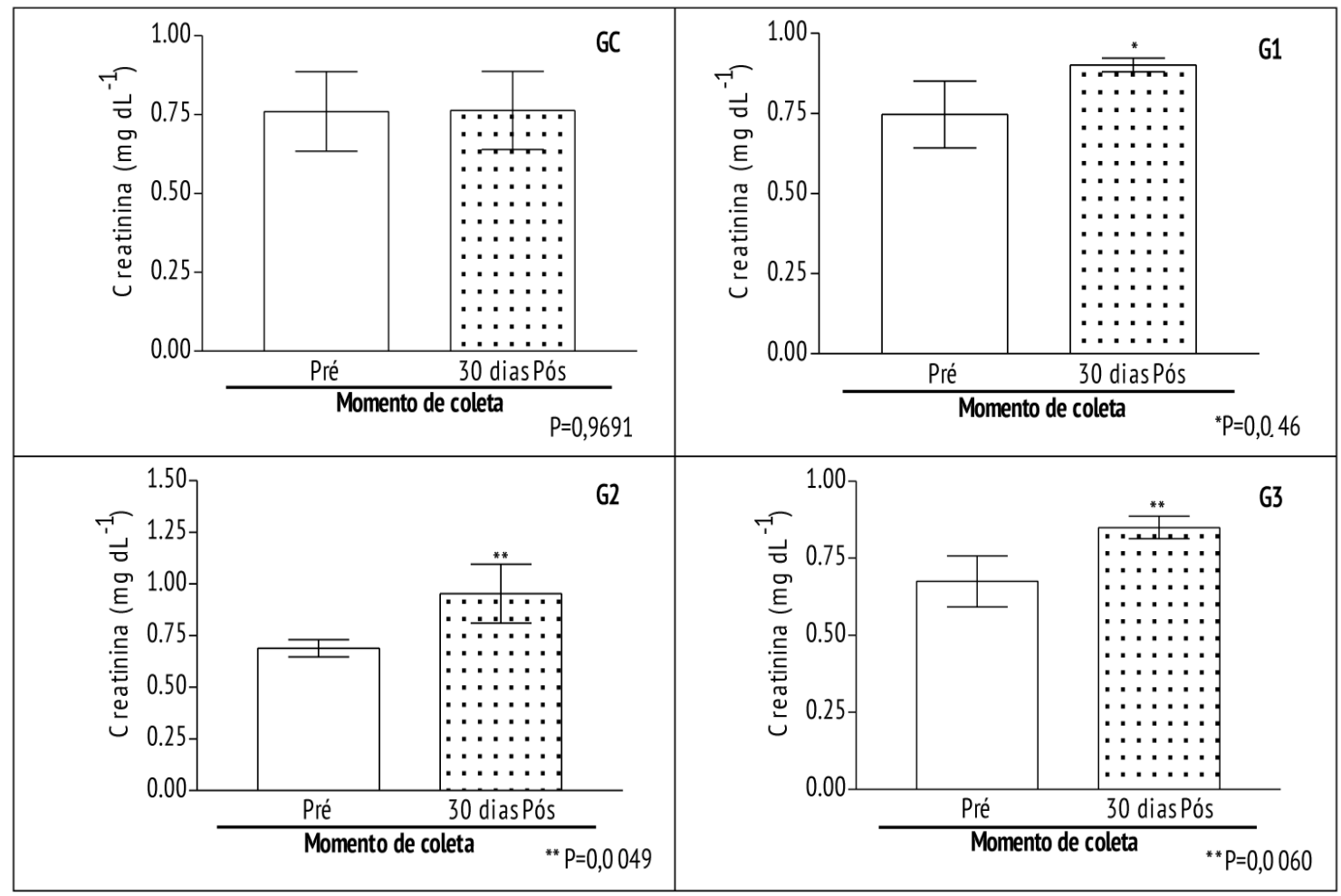

Figura 1 - Concentrações plasmáticas de creatinina $\left(\mathrm{mg}^{\mathrm{dL}} \mathrm{dL}^{-1}\right)$ no momento pré-operatório imediato e 30 dias após 30 minutos de isquemia quente em ratos Wistar, pertencentes aos grupos GC - grupo controle (rim esquerdo submetido à isquemia quente por 30 minutos, sem suplementação de AGPI n-3), G1 (rim esquerdo submetido à isquemia quente por 30 minutos, com suplementação de AGPI n-3 por 30 dias pré-operatório), G2 (rim esquerdo submetido à isquemia quente por 30 minutos, com suplementação de AGPI n-3 por 30 dias pós-operatório) e G3 (rim esquerdo submetido à isquemia quente por 30 minutos, com suplementação de AGPI n-3 por 30 dias pré e 30 dias pós-operatório.) Os dados estão apresentados como média \pm desvio padrão.

Tabela 2 - Tabulação das médias e desvio-padrão e do valor de p considerando-se as concentrações plasmáticas de creatinina pré e pós operatórias em cada grupo analisado no momento 30 dias pós-IQR

\begin{tabular}{|c|c|c|c|c|c|}
\hline \multicolumn{2}{|c|}{ Grupos/Momentos } & GC & G1 & G2 & G3 \\
\hline \multirow{2}{*}{$\begin{array}{l}\text { Creatinina } \\
\text { Média } \\
\left(\mathrm{mg} \cdot \mathrm{dL}^{-1}\right)\end{array}$} & Pré & $0,7600 \pm 0,1262$ & $0,7467 \pm 0,1046$ & $0,6883 \pm 0,04167$ & $0,6750 \pm 0,08264$ \\
\hline & Pós & $0,7633 \pm 0,1240$ & $0,9017 \pm 0,05193$ & $0,9533 \pm 0,1428$ & $0,8500 \pm 0,03633$ \\
\hline \multicolumn{2}{|c|}{ Valor de $p$} & 0,9691 & $0,046^{*}$ & $0,0049^{* *}$ & $0,0080^{* *}$ \\
\hline
\end{tabular}

Nota: ${ }^{*} p<0,05 ;{ }^{* *} p<0,01$.

GC - rim esquerdo submetido à isquemia quente por 30 minutos, sem suplementação de AGPI n-3.G1 - rim esquerdo submetido à isquemia quente por 30 minutos, com suplementação de AGPI n-3 por 30 dias pré-operatório. G2 - rim esquerdo submetido à isquemia quente por 30 minutos, com suplementação de AGPI n-3 por 30 dias pós-operatório. G3 - rim esquerdo submetido à isquemia quente por 30 minutos, com suplementação de AGPI n-3 por 30 dias pré e 30 dias pós-operatório. 


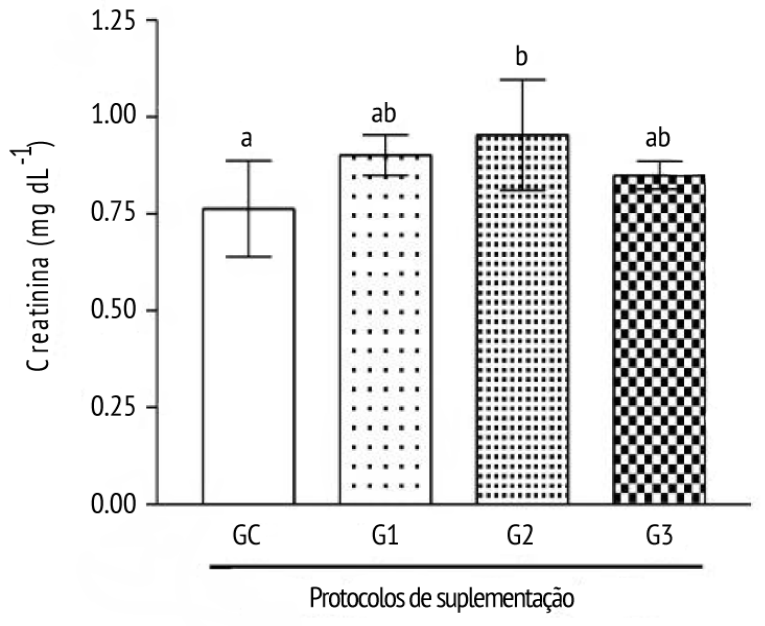

Figura 2 - Concentrações plasmáticas de creatinina $\left(\mathrm{mg}^{\mathrm{dL}}{ }^{-1}\right)$ no momento 30 dias após IQR por 30 minutos, entre os grupos GC - grupo controle (rim esquerdo submetido à isquemia quente por 30 minutos, sem suplementação de AGPI n-3), G1 (rim esquerdo submetido à isquemia quente por 30 minutos, com suplementação de AGPI n-3 por 30 dias pré-operatório), G2 (rim esquerdo submetido à isquemia quente por 30 minutos, com suplementação de AGPI n-3 por 30 dias pós-operatório) e G3 (rim esquerdo submetido à isquemia quente por 30 minutos, com suplementação de AGPI n-3 por 30 dias pré e 30 dias pósoperatório). Os dados estão apresentados como média \pm desvio padrão. Para letras iguais NS, para letras diferentes $p<0,05$.

\section{Discussão}

Neste estudo foi observado que $75 \%$ dos animais apresentaram aumento de creatinina compatível com IRA, segundo o critério RIFLE, no pós-IQR de 24 horas, independentemente do grupo avaliado. Este número reflete o impacto da lesão de isquemia e reperfusão e redução abrupta da massa renal (nefrectomia) em todos os animais em 24 horas, independentemente de suplementação prévia recebida (G1 e G3).

Neste período de 24 horas pós-IQR, o aumento de creatinina é esperado pois trata-se de fase de extensão, pós fase de iniciação da injúria, e pode durar de minutos até sete dias (Padanilam, 2003). É nesta fase de extensão que as lesões de reperfusão e inflamação ocorrem após o insulto isquêmico (Padanilam, 2003), e na dependência da extensão da

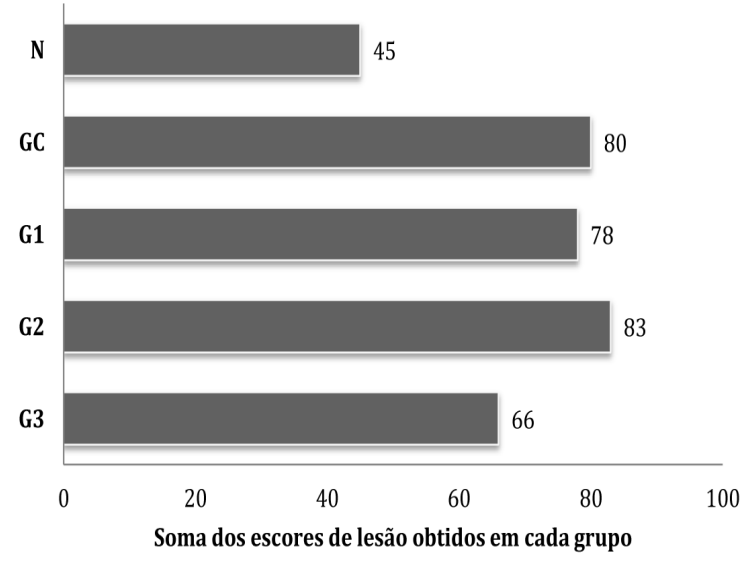

Figura 3 - Pontuação global dos escores de lesão histopatológica em cada grupo, no momento 30 dias pós-operatório, considerando-se $\mathrm{N}$-grupo normal (rim direito sem isquemia e sem suplementação), GC - grupo controle (rim esquerdo submetido à isquemia quente por 30 minutos, sem suplementação de AGPI n-3), G1 (rim esquerdo submetido à isquemia quente por 30 minutos, com suplementação de AGPI n-3 por 30 dias préoperatório), G2 (rim esquerdo submetido à isquemia quente por 30 minutos, com suplementação de AGPI n-3 por 30 dias pósoperatório) e G3 (rim esquerdo submetido à isquemia quente por 30 minutos, com suplementação de AGPI n-3 por 30 dias pré e 30 dias pós-operatório).

lesão epitelial é possível observar ou não respostas terapêuticas (Bonventre e Yang, 2011).

As variações de creatinina observadas aos 30 dias pós-IQR revelaram que os grupos suplementados apresentaram creatinina pós-IQR superiores e diferentes do momento pré-IQR quando comparadas ao grupo controle. Ainda, comparando-se apenas creatina pós-IQR, o grupo suplementado pós-IQR (G2) apresentou diferença em relação ao grupo controle, revelando que o uso do AGPI n-3, (EPA:DHA de 2:1) pode ter influenciado negativamente a recuperação da taxa de filtração nos animais suplementados e observados por 30 dias.

Sabe-se que toda reação inflamatória após injúria aguda será seguida de uma fase de reparação tissular. Questiona-se, neste momento, se a ação anti-inflamatória do AGPI n-3 possa interferir de forma negativa nesta fase de reparação. Isto pode 
não ocorrer nas doenças crônicas, justamente por ser o fator inflamatório crônico implicado como principal fator de progressão em muitas destas doenças.

Neumayer et al. (1992) avaliaram a TFG (taxa de filtração glomerular), mensurada por clearance de insulina e de creatinina, em cães previamente suplementados com AGPI n-3 por seis semanas e submetidos à isquemia renal; houve queda da TFG no pós-isquemia imediato, mas melhora (sem normalização) em 72 horas nos animais suplementados. Esta melhora da TFG não foi observada nos animais do grupo controle deste estudo.

Hassan e Gronert (2009) observaram que ratos submetidos à isquemia quente por 30 minutos, suplementados previamente com AGPI-n3 por quatro semanas, não mostraram aumentos dos valores plasmáticos de creatinina no pós-isquemia de 24 horas, diferentemente deste estudo, mas em isquemia de 45 minutos houve aumento significativo dos valores de creatinina no mesmo período, quando comparados com grupo controle.

Quanto à análise histopatológica, não foi observada diferença nos escores de lesão entre os grupos tratados e nem mesmo entre os rins não isquemiados e não suplementados considerados normais. An et al. (2009) demonstraram que o uso de AGPI $n-3$ foi efetivo para reduzir o infiltrado inflamatório tubulointersticial avaliado pelo número de células mononucleares $/ \mathrm{mm} 2 \mathrm{em}$ lâmina, mas não avaliaram o escore de lesão tubular, como no presente estudo, portanto não é possível comparar os resultados por divergir da metodologia empregada.

Fassett et al. (2010) realizaram compilação de trabalhos realizados no período de 25 anos, nos quais utilizaram AGPI n-3, especialmente EPA e DHA, no tratamento de diversas doenças renais, agudas e crônicas, com mecanismos patogênicos diferentes (nefropatia por IgA, nefrite por lúpus, doença renal policística, glomerulopatia membranosa, nefrectomia 5/6, nefropatia tóxica induzida por gentamicina e ciclosporina, nefropatia diabética), e observaram que o papel dos AGPI-n3 permanece não esclarecido para as doenças renais. Estes autores discutem que a não padronização de métodos de estudo, as variações do número populacional da amostra, da proporção de EPA e DHA fornecida, da forma de suplementação escolhida, da dose utilizada e do tempo de condução dos estudos dificultam a comparação entre os resultados, inclusive a realização de metanálise. Para a conclusão definitiva sobre o uso do AGPI-n3 sobre as lesões renais sugere-se a realização de estudo amplo, aleatorizado e com controle clínico.

Acredita-se que a dificuldade em comparar os dados obtidos no presente estudo com a literatura atual deve-se ao fato de não haver outro estudo realizado com a mesma metodologia

\section{Conclusão}

Aos 30 dias após IQR, todos animais suplementados apresentaram elevação dos valores de creatinina plasmática, quando comparada à creatinina pré isquemia. Comparando os três grupos suplementados ao grupo controle, o grupo que recebeu após IQR suplementação de 30 dias apresentou creatinina plasmática superior no pósoperatório de 30 dias.

Não foi observada diferença estatística nos escores de lesão histopatológica entre os grupos. Conclui-se que, nas condições propostas, o uso de ácido graxo poli-insaturado ômega 3 em todos os grupos suplementados não evitou o aumento de creatinina plasmática após isquemia quente renal, preservando, entretanto, a morfologia renal.

\section{Referências}

Abdeldaeim HM, Youssif TMA, Wahab MMA, Kotb AF, El Gebaly OF, Mokhless IA. Prospective randomized comparison between cold and warm ischemia in patients with renal insufficiency undergoing partial nephrectomy. Urology. 2015;85(4):862-8.

Adin CA, Scansen BA. Complications of upper urinary tract surgery in companion animals. Vet Clin North Am Small Anim Pract. 2011;41(5):869-88.

An WS, Kim HJ, Cho KH, Vaziri ND. Omega-3 fatty acid supplementation attenuates oxidative stress, inflammation, and tubulointerstitial fibrosis in 
the remnant kidney. Am J Physiol Renal Physiol. 2009;297(4):F895-903.

Arab-Tehrany E, Jacquot M, Gaiani C, Imran M, Desobry $S$, Linder M. Beneficial effects and oxidative stability of omega-3 long-chain polyunsaturated fatty acids. Trends Food Sci Tech. 2012;25(1):24-33.

Branco ACSC, Diniz MFFM, Almeida RN, Santos HB, Oliveira KM, Ramalho JA, et al. Parâmetros bioquímicos e hematológicos de ratos wistar e camundongos swiss do biotério professor Thomas George. R Bras Ci Saúde. 2011;15(2):209-14.

Bonventre JV, Yang L. Cellular pathophysiology of ischemic acute kidney injury. J Clin Invest. 201;121(11):4210-21.

Clark W, Parbtani A, Philbrick DJ, Holub BJ, Huff MW. Chronic effects of $\omega 3$ fatty acids (fish oil) in a rat 5/6 renal ablation model. J Am Soc Nephrol. 1991;1(12):1343-53.

Chertow GM, Burdick E, Honour M, Bonventre JV, Bates DW. Acute kidney injury, mortality, length of stay, and costs in hospitalized patients. J Am Soc Nephrol. 2005;16(11):3365-70.

De Caterina R, Caprioli R, Giannessi D, Sicari R, Galli C, Lazzerini $G$, et al. n-3 fatty acids reduce proteinuria in patients with chronic glomerular disease. Kidney Int. 1993;44(4):843-50.

Devarajan P. Update on mechanisms of ischemic acute kidney injury. J Am Soc Nephrol. 2006;17(6):1503-20.

Earley A, Miskulin D, Edmund JL, Levey AS, Uhlig K. Estimating equations for glomerular filtration rate in the era of creatinine standardization: a systematic review. Ann Intern Med. 2012;156(11):785-95.

Evora PRB, Pearson PJ, Seccombe JF, Schaff HV. Lesão de isquemia-reperfusão. Aspectos fisiopatológicos e a importância da função endotelial. Arq Bras Cardiol. 1996;66(4): 239-45.

Fassett RG, Gobe GC, Peake JM, Coombes JS. Omega-3 polyunsatured fatty acids in the treatment of kidney disease. Am J Kidney Dis. 2010;56(4): 728-42.
Goujon JM, Hauet T, Menet E, Levillain P, Babin P, Carretier M. Histological evaluation of proximal tubule cell injury in isolated perfused pig kidney exposed to cold ischemia. J Surg Res. 1999;82(2):228-33.

Halazun KJ, Al-Mukhtar A, Aldouri A, Willis S, Ahmad N. Warm ischemia in transplantation: search for a consensus definition. Transplant Proc. 2007;39(5):1329-31.

Hassan IR, Gronert K. Acute changes in dietary omega-3 and omega- 6 polyunsaturated fatty acids have a pronounced impact on survival following ischemic renal injury and formation of renoprotective docosahexaenoic acid-derived protectin D1. J Immunol. 2009;182(5):3223-32.

Hellegering J, Visser J, Kloke KJ, D’Ancona FCH, Hoitsma AJ, van der Vliet JA, et al. Deleterious influence of prolonged warm ischemia in living donor kidney transplantation. Transplant Proc. 2012;44(5):1222-6.

Holm T, Andreassen AK, Aukrust P, Andersen K, Geiran OR, Kjekshus J, et al. Omega-3 fatty acids improve blood pressure control and preserve renal function in hypertensive heart transplant recipients. Eur Heart J. 2001;22(5):428-36.

Holt PE. Outras causas de hematúria. In: Holt PE. Urologia em Cães e Gatos - Exames, diagnóstico e tratamento. $1^{\circ}$ ed. Rio de Janeiro: Revinter; 2011. p.123-33.

Hong S, Lu Y. Omega-3 fatty acid-derived resolvins and protectins in inflammation resolution and leukocyte functions: targeting novel lipid mediator pathways in mitigation of acute kidney injury. Front Immunol. 2013;4:13

Lauretani F, Maggio M, Pizzarelli F, Michelassi S, Ruggiero C, Ceda GP, et al. Omega-3 and renal function in older adults. Curr Pharm Des. 2009;15(36):4149-56.

Liu JC, Conklin SM, Manuck SB, Yao JK, Muldoon MF. Long-chain omega 3 fatty acids and blood pressure. Am J Hypertens. 2011;24(10):1121-6.

Malek M, Nematbakhsh M. Renal ischemia/reperfusion injury; from pathophysiology to treatment. J Renal Inj Prev. 2015;4(2):20-7. 
Neumayer HH, Heinrich M, Schimssas M, Haller H, Wagner $\mathrm{K}$, Luft FC. Amelioration of ischemic acute renal failure by dietary fish oil administration in conscious dogs. J Am Soc Nephrol. 1992;3(6):1312-20.

Nolan CR, Anderson RJ. Hospital-acquired acute renal failure. J Am Soc Nephrol. 1998;9(4):710-8.

Padanilam BJ. Cell death induced by acute renal injury: a perspective on the contributions of apoptosis and necrosis. Am J Physiol Renal Physiol. 2003;284(4):F608-27.

Pickering JW, Endre ZH. The definition and detection of acute kidney injury. J Renal Inj Prev. 2013;3(1):21-5.

Ricci Z, Cruz D, Ronco C. The RIFLE criteria and mortality in acute kidney injury: a systematic review. Kidney Int. 2008;73(5):538-46.

Ronco C. Acute kidney injury: from clinical to molecular diagnosis. Crit Care. 2016;20(1):201.

Siedlecki A, Irish W, Brennam DC. Delayed graft function in the kidney transplant. Am J Transplant. 2011;11(11):2279-96.

Simmons MN, Fergany AF, Campbell SC. Effect of parenchymal volume preservation on kidney function after partial nephrectomy. J Urol. 2011;186(2):405-10.

Tennankore KK, Kim SJ, Alwayn IP, Kiberd BA. Prolonged warm ischemia time is associated with graft failure and mortality after kidney transplantation. Kidney Int. 2016;89(3):648-58.

Thompson RH, Lane BR, Lohse CM, Leibovich BC, Fergany A, Frank I, et al. Every minute counts when the renal hilum is clamped during partial nephrectomy. Eur Urol. 2010;58(3):340-5.

Thompson RH, Lane BR, Lohse CM, Leibovich BC, Fergany A, Frank I, et al. Renal function after partial nephrectomy: effect of warm ischemia relative to quantity and quality of preserved kidney. Urology. 2012;79(2):356-60.

Vaidya VS, Ferguson MA, Bonventre JV. Biomarkers of acute kidney injury. Annu Rev Pharmacol Toxicol. 2008;48:463-93.
Volpe A, Blute ML, Ficarra V, Gill IS, Kutikov A, Porpiglia $F$, et al. Renal ischemia and function after partial nephrectomy: a collaborative review of the literature. Eur Urol. 2015;68(1):61-74.

Waitzberg DL. Ômega-3: o que existe de concreto? São Paulo: Nutrilite. 2007;1-38. [acesso em 11 Julho 2012]. Disponível em: https://tinyurl.com/j84hmf2.

Woodman RJ, Mori TA, Burke V, Puddey IB, Barden A, Watts GF, et al. Effects of purified eicosapentaenoic acid and docosahexaenoic acid on platelet, fibrinolytic and vascular function in hypertensive type 2 diabetic patients. Atherosclerosis. 2003;166(1):85-93.

Yaqoob P. The nutritional significance of lipid rafts. Annu Rev Nutr. 2009;29:257-82.
Recebido em: 19/01/2017

Received in: 01/19/2017

Aprovado em: 30/01/2017

Approved in: 01/30/2017 